\title{
Clinical guidelines on perioperative management strategies for enhanced recovery after lung surgery
}

\author{
Shugeng Gao ${ }^{1}$, Serena Barello ${ }^{2}$, Liang Chen ${ }^{3}$, Chun Chen ${ }^{4}$, Guowei Che ${ }^{5}$, Kaican Cai ${ }^{6}$, Roberto Crisci ${ }^{7}$, \\ Antonio D'Andrilli ${ }^{8}$, Andrea Droghetti ${ }^{9}$, Xiangning Fu ${ }^{10}$, Paolo Albino Ferrari ${ }^{11}$, Hiran C. Fernando ${ }^{12}$, \\ Di Ge ${ }^{13}$, Guendalina Graffigna ${ }^{2}$, Yunchao Huang ${ }^{14}$, Jian Hu${ }^{15}$, Wenjie Jiao ${ }^{16}$, Gening Jiang ${ }^{17}$, Xiaofei Li $^{18}$, \\ Hui $\mathrm{Li}^{19}$, Shanqing $\mathrm{Li}^{20}$, Lunxu Liu ${ }^{5}$, Haitao $\mathrm{Ma}^{21}$, Dongchun $\mathrm{Ma}^{22}$, Guillermo Martinez ${ }^{23}$, Giulio Maurizi ${ }^{8}$, \\ Kevin Phan ${ }^{24}$, Kun Qiao ${ }^{25}$, Majed Refai ${ }^{26}$, Erino A. Rendina ${ }^{27}$, Guoguang Shao ${ }^{28}$, Jianfei Shen ${ }^{29}$, \\ Hui Tian $^{30}$, Luca Voltolini ${ }^{31}$, Jacopo Vannucci ${ }^{32}$, Camilla Vanni ${ }^{8}$, Qingchen $\mathbf{W u}^{33}$, Shidong Xu ${ }^{34}$, Fenglei Yu ${ }^{35}$, \\ Song Zhao ${ }^{36}$, Peng Zhang ${ }^{37}$, Lanjun Zhang ${ }^{38}$, Xiuyi Zhi ${ }^{39}$, Chengchu Zhu ${ }^{29}$, Calvin Ng ${ }^{40}$, Alan D. L. Sihoe ${ }^{41}$, \\ Anthony M. H. Ho ${ }^{42}$
}

${ }^{1}$ Department of Thoracic Surgery, Cancer Institute \& Hospital, Chinese Academy of Medical Sciences, Beijing 100730, China; ${ }^{2}$ Department of Psychology, EngageMinds Hub Research Center, Università Cattolica del Sacro Cuore, Milan, Italy; ${ }^{3}$ Department of Thoracic Surgery, The First Affiliated Hospital of Nanjing Medical University, Nanjing 211166, China; ${ }^{4}$ Department of Thoracic Surgery, Fujian Medical University Union Hospital, Fuzhou 350122, China; ${ }^{5}$ Department of Thoracic Surgery, West China Hospital, Sichuan University, Chengdu 610065, China; ${ }^{6}$ Department of Thoracic Surgery, Nanfang Hospital, Southern Medical University, Guangzhou 510515, China; ${ }^{7}$ Division of Thoracic Surgery, University of L'Aquila, Mazzini Hospital, Teramo, Italy; ${ }^{8}$ Department of Thoracic Surgery, Sant'Andrea Hospital, Sapienza University of Rome, Rome, Italy; ${ }^{9}$ Division of Thoracic Surgery, Carlo Poma Hospital, Mantova, Italy; ${ }^{10}$ Department of Thoracic Surgery, Tongji Hospital, Tongji Medical College, Huazhong University of Science and Technology, Wuhan 430030, China; ${ }^{11}$ Division of Thoracic Surgery, A. Businco Cancer Center, Azienda Ospedaliera Brotzu, Cagliari, Italy; ${ }^{12}$ Inova Cardiac and Thoracic Surgery, Department of Surgery, Inova Fairfax Medical Campus, Falls Church, Virginia, USA; ${ }^{13}$ Department of Thoracic Surgery, Zhongshan Hospital of Fudan University, Shanghai 200032, China; ${ }^{14}$ Department of Thoracic Surgery, Cancer Research Institute of Yunnan Province, The Third Affiliated Hospital of Kunming Medical University (Yunnan Cancer Hospital), Kunming 650106, China; ${ }^{15}$ Department of Thoracic Surgery, The First Affiliated Hospital, Zhejiang University, Hangzhou 310003 , China; ${ }^{16}$ Division of Thoracic Surgery, The Affiliated Hospital of Qingdao University, Qingdao 266555, China; ${ }^{17}$ Department of Thoracic Surgery, Shanghai Pulmonary Hospital, Tongji University, Shanghai 200433, China; ${ }^{18}$ Department of Thoracic Surgery, Tangdu Hospital, The Fourth Military Medical University, Xi'an 710038, China; ${ }^{19}$ Department of Thoracic Surgery, Beijing Chao-Yang Hospital, Capital Medical University, Beijing 100020, China; ${ }^{20}$ Department of Thoracic Surgery, Peking Union Medical College Hospital, CAMS \& PUMC, Beijing 100032, China; ${ }^{21}$ Department of Thoracic Surgery, The First Affiliated Hospital of Soochow University, Soochow 215006, China; ${ }^{22}$ Department of Cardiothoracic Surgery, Anhui Chest Hospital, Hefei 230022, China; ${ }^{23}$ Department of Anesthesia and Intensive Care, Papworth Hospital, Cambridge, UK; ${ }^{24}$ Collaborative Research (CORE) Group, Macquarie University, Sydney, Australia; ${ }^{25}$ Department of Thoracic Surgery, The Third People's Hospital of Shenzhen, Shenzhen 518034, China; ${ }^{26}$ Division of Thoracic Surgery, AOU Ospedali Riuniti, Ancona, Italy; ${ }^{27}$ Department of Thoracic Surgery, Sant'Andrea Hospital, Sapienza University of Rome, Rome, Italy; ${ }^{28}$ Department of Thoracic Surgery, The First Hospital of Jilin University, Changchun 130021 , China; ${ }^{29}$ Department of Thoracic Surgery, Taizhou Hospital of Zhejiang Province, Wenzhou Medical University, Wenzhou 325035, China; ${ }^{30}$ Department of Thoracic Surgery, The Qilu Hospital of Shandong University, Jinan 250012, China; ${ }^{31}$ Thoracic Surgery Unit, Careggi University Hospital, Florence, Italy; ${ }^{32}$ Department of Thoracic Surgery, University of Rome Sapienza, Policlinico Umberto I, Rome, Italy; ${ }^{33}$ Department of Cardiothoracic Surgery, The First Affiliated Hospital of Chongqing Medical University, Chongqing 400042, China; ${ }^{34}$ Department of Thoracic Surgery, Harbin Medical University Cancer Hospital, Harbin 150081, China; ${ }^{35}$ Department of Thoracic Surgery, The Second Xiangya Hospital of Central South University, Changsha 410011, China; ${ }^{36}$ Department of Thoracic Surgery, The First Affiliated Hospital of Zhengzhou University, Zhengzhou 450052, China; ${ }^{37}$ Department of Cardiothoracic Surgery, Tianjing General Hospital of Tianjing Medical University, Tianjing 300052, China; ${ }^{38}$ Department of Thoracic Surgery, Sun Yat-sen University Cancer Center, Guangzhou 510060, China; ${ }^{39}$ Department of Thoracic Surgery, Xuanwu Hospital of Capital Medical University, Beijing 100053, China; ${ }^{40}$ Division of Cardiothoracic Surgery, Department of Surgery, The Chinese University of Hong Kong, Prince of Wales Hospital, Hong Kong SAR, China; ${ }^{41}$ Gleneagles Hong Kong Hospital, Hong Kong, China; ${ }^{42}$ Department of Anesthesiology and Perioperative Medicine, Queen's University Kingston, Ontario, Canada 
Correspondence to: Shugeng Gao. Department of Thoracic Surgery, Cancer Institute \& Hospital, Chinese Academy of Medical Sciences, Beijing 100021, China. Email: gaoshugeng@vip.sina.com.

Submitted Oct 02, 2019. Accepted for publication Dec 20, 2019.

doi: $10.21037 /$ tlcr.2019.12.25

View this article at: http://dx.doi.org/10.21037/tlcr.2019.12.25

\section{Introduction}

The concept of enhanced recovery after surgery (ERAS) was first developed in Denmark in 1997 by Dr. Kehlet (1). ERAS is designed to optimize perioperative management, improve patient prognosis, reduce complications, shorten hospital stay, and lower cost (2-5). In recent years, this multi-disciplinary and multi-modal perioperative rehabilitation concept has been widely applied in open and endoscopic procedures including colorectal surgery $(6,7)$, gynecological surgery $(8,9)$, liver surgery $(10,11)$, breast surgery $(12,13)$, urologic surgery $(14,15)$, and spinal surgery (16-18).

Advances in thoracic surgery, especially the popularization of minimally invasive techniques, have dramatically expanded or changed the concept of surgical treatment (19-21). Early recovery from thoracic surgery is now routine (22-24). In Jan 2019, the European Society of Thoracic Surgeons published guidelines for enhanced recovery after lung surgery (25) on: preoperative counselling, nutritional screening, smoking cessation, rehabilitation for high-risk patients, avoidance of fasting, carbohydrate loading, avoidance of preoperative sedatives, venous thromboembolism prophylaxis, prevention of hypothermia, short-acting anesthetics to facilitate early emergence, regional anesthesia, nausea and vomiting control, opioid-sparing analgesia, euvolemic fluid management, minimally invasive surgery, early chest drain removal, avoidance of urinary catheters, and early mobilization after surgery.

The lung is a unique organ in that it receives the total cardiac output and acts as a giant filter for systemic venous blood (26). It is, in addition, an open organ; through the airway, the alveoli interact with the surrounding environment. Due to its unique anatomy and physiology, the lung is susceptible to injuries caused by a variety of harmful endogenous and exogenous factors (27). Perioperative risk factors and treatment measures can result in damage to the lungs, which in turn can lead to postoperative pulmonary complications (PPCs) (e.g., atelectasis and pneumonia) and pleural complications, affecting early and long-term recovery (28-30), and accounting for up to $84 \%$ of all deaths (31). Perioperative airway and protective ventilatory management in lung surgery are an important part of ERAS $(32,33)$. Given the circumstances, we have established clinical guidelines based primarily on an extensive literature review, with discussion and consensus focused on the issue of perioperative airway management for enhanced recovery following lung surgery. In addition, we will briefly review other perioperative measures designed toward the goal of ERAS for thoracic surgery.

\section{Preoperative preparations}

\section{Patient education}

The content of preoperative patient education includes the patient's self-preparation, introduction to relevant policies, postoperative bed arrangement, postoperative cooperation, postoperative symptom management, and individualized guidance (34). Patient education helps patients to better understand their disease, the objectives behind their treatment, and the nursing care they will receive. It also supports patients in dealing with the psychological impact of their disease and helps them to adapt to life as an inpatient. Furthermore, it encourages patients' cooperation throughout their treatment, including at the surgery and postoperative rehabilitation stages. It also enables patients to master methods and techniques for improving lung function and promoting lung rehabilitation, builds their confidence in surgery, and promotes self-care awareness and postoperative rehabilitation. Both patient satisfaction and the patient experience in healthcare institutions are improved by patient education (35-37). In addition to verbal instructions, patients should be provided with written and multimedia material illustrating care plans.

\section{Patient engagement}

Patient engagement directly impacts outcomes such as patient adherence, patient self-management, preventing behaviors, satisfaction with care, and lower health care costs. The Patient Health Engagement model 
$\left(\right.$ PHE-S $\left.^{\circledR}\right)$ - which can identify and monitor patients' level of psychological readiness to be an active player in their healthcare team - could be a reliable instrument to facilitate and support the active engagement of patients in their own care (38-40).

\section{Smoking cessation}

The relative risk of PPCs in smokers is 1.4 to 4.3 times that of nonsmokers. Even in patients without chronic lung disease, smoking is a risk factor for increased PPCs (41). Quitting smoking is one of the most effective ways to prevent PPCs. It has been found that if patients quit smoking for more than 4 weeks before surgery, PPCs can be reduced (42). However, in patients with a smoking index of $\geq 800$ cigarette-years, smoking cessation even 2 weeks before surgery will not lower the risk of PPCs $(43,44)$. Compared with non-smokers, smokers have significantly longer hospital stays after lung surgery, along with significantly higher mortality (45).

\section{Preoperative pulmonary function assessment}

The pulmonary function test (PFT) is affected by ventilation and airway obstruction $(46,47)$. It helps the surgeon to comprehend the nature, severity, and reversibility of the lung disease and thus facilitates the prediction of surgical outcomes and pulmonary complications, as well as the selection of the appropriate type of surgery and its extent (48). The forced expiratory volume at $1 \mathrm{sec}$ (FEV1) can independently predict the risk of lung resection. Patients with abnormal results of PFT (especially low FEV1) are at a higher risk for PPCs. These patients should undergo adequate preoperative examinations to identify the surgical risks and appropriate measures that need to be taken to improve the patient's lung function and decrease the risk of PPCs (49-52). Some patients may benefit from optimization of lung function by pulmonary physician. The peak expiratory flow (PEF)—also called peak expiratory flow rate (PEFR) - refers to the maximal rate that a person can exhale during a short maximal expiratory effort. It mainly reflects the strength of the respiratory muscles and the patency of the airway but may also reflect the ability to cough, which is highly effort-dependent. Decreased PEF is seen in patients with obstructive or restrictive ventilatory disorders. If PEF is $<320 \mathrm{~L} / \mathrm{min}$, the patient can have trouble expelling phlegm after surgery, which may result in lung infection (29).

\section{Preoperative airway management}

For high-risk patients, airway preparation, including drug therapy combined with physical rehabilitation, should be performed before surgery. Commonly used drugs include antibiotics, glucocorticoids, bronchodilators, and mucolytic agents. The combinations of nebulized drugs (e.g., budesonide inhalation suspension + terbutaline sulfate solution for inhalation + acetylcysteine solution for inhalation) help to reduce airway hyperresponsiveness and prevent perioperative airway complications (53-55).

The recommended physical rehabilitation measureswhich include chest physiotherapy and incentive spirometry, climbing stairs, and power-based cycling-help to increase lung capacity and improve lung function (56). The intensity and duration of training are based patient-dependent. They should not increase the patient's disease burden but should be sufficient for the patient to gain the benefits of regular exercise. Besides FEV1, the diffusion capacity of carbon monoxide in unit time (DLCO) is also important and when levels are low, CPET (cardiopulmonary exercise testing) evaluation should be carried out to identify those who require rehabilitation.

\section{Preoperative anti-infective treatment}

Pulmonary bacterial infections should be treated rationally with antibiotics. Elective surgery should be postponed until the acute respiratory infection is resolved. For patients with excessive sputum production, surgery should not be performed until 2 weeks after the sputum volume is reduced. For patients with accompanying chronic respiratory diseases, antibiotics can be used 3 days before surgery (57). In patients with a history of heavy smoking or moderate to severe emphysema, pathogenic bacteria may colonize mouth, pharynx, and upper and lower respiratory tracts before surgery, which can increase the incidence of postoperative pneumonia. Perioperative antibiotic prophylaxis can reduce pulmonary complications.

\section{Intraoperative management}

\section{Anesthesia}

The selection of anesthesia method and drugs should be made according to the following principles: effectiveness in sedation, analgesia, and muscle relaxation; rapid postoperative awakening and recovery; satisfactory blockage of adverse nerve reflex after surgery; small anesthesia- 
induced trauma and few disturbances to respiration/ circulation; and fewer complications $(20,58,59)$.

During the operation, target-oriented personalized volume management is the best way to reduce postoperative acute lung injury $(33,60)$. Circulation should be kept stable, and high or low blood pressure should be avoided. Correctable causes of arrhythmia, if present, should be identified. The airway must be kept patent ensure proper ventilation $(61,62)$. Intraoperative application of bronchodilators can reduce bronchospasm (63-65).

Thoracic surgery usually requires a double-lumen endotracheal tube for one-lung ventilation. The goal of one-lung ventilation is to ensure adequate surgical exposure while maintaining adequate oxygenation, and to avoid acute lung injury $(66,67)$. At present, lung-protective ventilation strategies comprise: low tidal volume $(4-6 \mathrm{~mL} / \mathrm{kg})$, positive end-expiratory pressure (PEEP), and lung recruitment (68). Among them, low tidal volume is the most important mode, and tidal volume and PEEP should be dynamically adjusted according to the patient's respiratory mechanics. Lung recruitment refers to the process of reopening the atelectatic lung unit by increasing the transpulmonary pressure. At present, PEEP titration is recommended to replace lung recruitment maneuvers. In addition, low-tomoderate inhaled oxygen concentrations $\left(\mathrm{FiO}_{2}, 30 \%\right.$ to $50 \%)$ should be used when satisfactory oxygen saturation is achieved (69).

\section{Surgical management}

Careful preoperative planning optimizes the surgical procedure and thus helps to shorten the operative time and reduce surgical trauma (24,70). Minimally invasive techniques are recommended; in particular using incisions that limit muscle trauma and reduce postoperative pain are preferred (71). In an open approach, muscle-sparing thoracotomy (preserving the latissimus dorsi and serratus anterior muscles) may be considered as an alternative to posterolateral thoracotomy. Excessive stretching, squeezing, and/or twisting of lung tissue must be avoided. Two "maximizations" should be achieved during the surgical treatment of lung cancer: maximization of the removal of the tumor and maximization of the preservation of the lung tissue (72). The integrity of the thoracic cage also needs to be maintained and major nerves such as the recurrent laryngeal nerve, phrenic nerve, and vagus nerve should be protected (73). Preservation of the long thoracic or accessory nerves is also important. Care must also be taken to prevent and reduce the occurrence of pulmonary air leaks (74). The negative venous pressure should be carefully managed to prevent air embolism (75). To avoid fat embolism, treatment of fractures should be gentle (76).

\section{Postoperative treatment}

\section{Postoperative airway management and pulmonary rebabilitation}

Aerosol inhalation should be initiated early after surgery to humidify the airway and be directly applied to the airway mucosa (77). The combination of glucocorticoids (e.g., budesonide inhalation suspension $2 \mathrm{mg}$ per time, 2-4 times per day) with bronchodilators (e.g., terbutaline sulfate inhalation solution, $5 \mathrm{mg}$ per time, $2-4$ times per day) relieves airway inflammation (78). Active coughing and expectoration should be encouraged (79); meanwhile, mucolytics (e.g., acetylcysteine inhalation solution, $300 \mathrm{mg}$ per dose, 2 times per day) can dissolve and dilute the mucous sputum in the airway, thus facilitating expectoration and reducing the risk of postoperative complications (80). In addition, it has been found that multidisciplinary collaborative airway management strategies including incentive spirometry, coughing and deep breathing, oral health care, patient (and family) education, early and ambulation at least 3 times daily, and raising the head of bed (by $\geq 30^{\circ}$ ) can substantially reduce the incidences of pneumonia and unplanned intubation in the postoperative period $(81,82)$.

\section{Rational use of analgesics}

Along with physical rehabilitation, postoperative pain management plays a key role in achieving the rapid improvement of lung function $(83,84)$. Effective postoperative analgesic measures can promote early diaphragmatic movement, coughing, and expectoration, thereby reducing damage to lung function and decreasing pulmonary infections (85). According to the ERAS principles, opioids should be avoided if possible, and epidural, paravertebral, or erector spinae plane anesthesia with ropivacaine with or without an opioid is recommended. Both are superior to intravenous morphine and can significantly improve postoperative lung function (86) without central nervous depression. Analgesia by means of other techniques such as transcutaneous electrical nerve stimulation (TENS) or serratus anterior plane (SAP) block may be used as a technical adjunct to control severe 
pain (87-90). Furthermore, pain can be alleviated by early removal of chest drain (91).

\section{Early ambulation}

In 1949, Leithauser acknowledged that early ambulation was essential for the well-being and safety of patients undergoing surgery (92). In addition, he proposed that early mobilization can save lives by avoiding protracted hospitalization, thus preventing many fatal complications, and that rehabilitation exercise early after surgery is an important means to prevent PPCs $(93,94)$. To achieve early ambulation, postural adjustments and shoulder movement can be increased (95). It was also found that using a pedal exerciser while sitting alone on or after the second postoperative day can significantly reduce the incidence of postoperative respiratory tract infections and dyspnea and significantly shorten hospital stays (96). Although there have been no confirmed differences between the exercise capacity or lung function of patients who commenced early postoperative physiotherapy protocol and those who started later, patients with operable cancer seem to benefit from early exercise programs in terms of fatigue reduction (97). However, the effect of an increased postoperative activity level in the early postoperative lung surgery must be further evaluated (98).

\section{Expert opinion}

How about the application of ERAS in the surgical treatment of lung cancer in your center? Has a treatment system been formed?

\section{Expert opinion 1: Alan D. L. Sihoe}

In my old hospital in Hong Kong, a program for patient care after lung cancer surgery was already used since the early 2000s. In 2007, to complement the increasing use of VATS, I designed a new Clinical Pathway for use with patients receiving lung cancer surgery by VATS (another way of saying 'fast track' or ERAS). It significantly improved our outcomes, but was still not good enough. In 2010, I further updated our hospital's Clinical Pathway to make it more detailed, more user-friendly, and more easily audited. This improved results further and allowed variance analyses to enhance quality assessment. In 2014, I transposed the Clinical Pathway for use in our new hospital in Shenzhen, and further adapted it to the needs of modern Uniportal VATS and incorporated advances such as routine use of digital chest drain technology. As you can see, we not only have a very long-established traditional of comprehensive ERAS management, but we have constantly improved it over time.

\section{Expert opinion 2: Andrea Droghetti}

Yes, we have. We are organizing with our administration the pathway in hospital.

\section{Expert opinion 3: Antonio D'Andrilli}

$E R A S$ application is not standardized for all patients, but most of ERAS recommendations are currently applied.

\section{Expert opinion 4: Calvin $\mathbf{N g}$}

Yes, we have a post-operative clinical pathway that we should follow unless clinical condition of the patient deviates from the expected, allowing early mobilization, targeted physiotherapy, optimization of pain-control and resumption of oral diet. We also have pre-operative clinic with the patients 2 weeks before surgery to educate them about the operation, what to expect, how to cooperate with physiotherapy during hospital stay, ensure smoking cessation, optimizing blood pressure and diabetes control, avoiding chest infections prior to surgery, improving their diet etc.

\section{Expert opinion 5: Luca Voltolini}

Yes, we have applied ERAS protocol to the surgical treatment of lung cancer by VATS.

\section{Expert opinion 6: Majed Refai}

ERAS is already adopted in my center. I began with TEAM education in 2017.

\section{Expert opinion 7: Paolo Ferrari}

In 2017 we started a FAST TRACK protocol assessment for surgical lung cancer management. After one year of experimental application, according to ERAS guidelines produced by Italian VATS Group, we introduced an enhanced recovery trail for all patients underwent VATS lobectomy for NSCLC.

\section{Expert opinion 8: Roberto Crisci}

In my center a course of treatment has been formed, however practical-administrative reasons have so far prevented the clinical start.

\section{Expert opinion 9: Anthony M. H. Ho}

Kingston General Hospital does not have a formal ERAS for thoracic surgery, but anesthesiologists already practice 
most or all of the principles of ERAS.

Indwelling urethral catheter is routinely used after surgery in most patients with lung cancer. For patients with normal renal function before surgery, should early removal of indwelling catheter be recommended to relieve postoperative discomfort?

Expert opinion 1: Alan D. L. Sihoe

I disagree. A urinary catheter is NOT routinely used in lung cancer surgery. A long time ago, we would insert a catheter only if we required accurate assessment of a patient's fluid balance during and after surgery (e.g., in pneumonectomy or complex operations). However, we have NOT used urinary catheters in any lung cancer surgery patients now for over 12 years.

\section{Expert opinion 2: Andrea Droghetti}

When we use the cath, we remove the day after surgery.

\section{Expert opinion 3: Antonio D'Andrilli}

Urethral catheter is generally not used in patients undergoing major lung resection in our Institution.

\section{Expert opinion 4: Calvin Ng}

We do not routinely place urinary catheters in our lung resection patients. Most straight forward major lung resection cases finish within 2-3 hours. For complicated cases with expected prolonged operating time, or cases with expected significant blood loss, or cases of patients with poor cardiac function we would place a catheter. Occasionally, patients without catheter have urinary retention in the post-anesthesia recovery room, and we would catheterize once (meaning catheterize to drain urine completely then remove) to relieve the retention.

\section{Expert opinion 5: Luca Voltolini}

Yes. Our protocol includes the removal of indwelling urethral catheter between 12 and 48 hours after surgery. Again, this is true for all VATS lobectomies in which we don't use the epidural catheter.

\section{Expert opinion 6: Majed Refai}

I agree that early removal is recommended.

\section{Expert opinion 7: Paolo Ferrari}

The indwelling urethral catheter should be avoided in patients without renal impairment or other urinary tract pathologies. We strongly recommend the discharge of the urethral catheter in the early postoperative, after a clamping test.

\section{Expert opinion 8: Roberto Crisci}

Yes.

\section{Expert opinion 9: Anthony M. H. Ho}

Foley catheters are removed routinely the next morning after surgery unless there is an epidural. For women with an epidural, Foley is removed 1-2 days after surgery. For men, Foley is removed 1-2 days after surgery but for elderly men with an epidural, it could be $2-3$ days after surgery to coincide with the withdrawal of epidural analgesia.

\section{Placement of indwelling chest tubes is associated with postoperative pain and can inbibit breathing. Is it feasible to use a single chest tube instead of two after routine anatomical pneumonectomy? When do you think of a single chest tube should be used and when should two chest tubes be used?}

\section{Expert opinion 1: Alan D. L. Sihoe}

We have not used 2 chest drains in any lung cancer surgery patients for over 15 years. In any standard lung cancer operation, I cannot see any situation where I would ever use 2 chest drains. (I only use 2 drains sometimes after complex empyema surgery-but that has nothing to do with lung cancer).

Expert opinion 2: Andrea Droghetti

We use always only one chest tube, never two.

\section{Expert opinion 3: Antonio D'Andrilli}

Single chest tube is used in most major lung resections at our Institution. Single tube is always used after minimally invasive procedures and after pneumonectomy.

\section{Expert opinion 4: Calvin $\mathrm{Ng}$}

In routine segmentectomy, lobectomy and pneumonectomy, we usually place only one chest drain. We may occasionally place 2 drains if we expect the post-operative drainage to be very heavily blood stained, but for pneumonectomy we always place one drain. For operations such as decortication for empyema, we would routinely place 2 drains.

\section{Expert opinion 5: Luca Voltolini}

Our ERAS program suggests using just one pleural drainage 
(28/30 Fr) after pulmonary lobectomy. We consider to place a second tube in case of bilobectomy, significant postoperative air leak (very rarely) or when a significant postoperative bleeding is expected (in case of coagulopathy or when an aggressive anticoagulation therapy is required).

\section{Expert opinion 6: Majed Refai}

Single chest tube is sufficient even after pneumonectomy. We use only single chest tube.

\section{Expert opinion 7: Paolo Ferrari}

In our Institution we mean pneumonectomy as a complete removal of an entire lung, where a single chest tube is suggested for a correct pressure balance inside the residual chest cavity, until the chest tube removal. Regarding anatomical lobectomy or segmentectomy, a single chest tube is recommended, possibly with the new concepts of draining system (spiral drain-smart drain). We reserve double chest drain for those procedures where an extensive pleural decortication or chest wall surgery are associated.

\section{Expert opinion 8: Roberto Crisci}

The current surgery always provides for the use of only one drain, except in exceptional cases.

\section{Expert opinion 9: Anthony M. H. Ho}

After open pneumonectomy, one chest drain is inserted. We don't do VATS for pneumonectomy in Kingston. Rarely ever have 2 chest drains.

\section{Some studies have shown that chest tube can be safely removed after surgery for lung cancer even if the amount of daily pleural fuid production is larger (up to $450 \mathrm{~mL} / 24$ b). What are the routine criteria for chest tube removal in your center?}

\section{Expert opinion 1: Alan D. L. Sihoe}

In our ERAS protocol of 2010, our removal criteria are: (I) no air leak for 24 hours; (II) $200 \mathrm{~mL}$ over last 24 hours or less; (III) no evidence of pleural space infection. However, in our 2014 protocol, we have revised this to: (I) air flow on digital system $<40 \mathrm{~mL} / \mathrm{min}$ for 6 hours with no 'spikes'; (II) 200-300 mL over last 24 hours (but this can be higher at the discretion of the surgeon); (III) no evidence of pleural space infection. This protocol is for my fellows/ residents to follow. But in reality, if I am doing the ward round myself, I often remove with even higher daily fluid outputs.

\section{Expert opinion 2: Andrea Droghetti \\ Under $300 \mathrm{~mL} / 24 \mathrm{~h}$.}

\section{Expert opinion 3: Antonio D'Andrilli}

Threshold generally used for chest tube removal at our Institution is $3-4 \mathrm{~mL} / \mathrm{kg}$.

\section{Expert opinion 4: Calvin $\mathrm{Ng}$}

In terms of volume of drainage, we use a figure of $200 \mathrm{mLs} / 24$ hours as the cut off for drain removal. I believe that perhaps the volume in realtion to body size or weight is a more sensible measurement. For example, $450 \mathrm{~mL} / 24 \mathrm{hrs}$ in a $50 \mathrm{~kg}$ patient is different to the same volume in a $100 \mathrm{~kg}$ patient. There are other considerations too, for example, if the drainage fluid is heavily blood stained then we would tend to keep the drain longer even if drainage volume is below $200 \mathrm{mLs} / 24 \mathrm{hrs}$. We also look at the drainage volume trend over the past few days, and the chest radiograph to see if we need to shift out the drain to release basal collection.

\section{Expert opinion 5: Luca Voltolini}

We remove the chest tube when the fluid drainage/day is less than $4-5 \mathrm{~mL} / \mathrm{kg}$, considering that it sits within the range of physiological daily pleural fluid filtration.

\section{Expert opinion 6: Majed Refai}

We remove chest tube if daily fluid production is less than $400 / 24 \mathrm{~h}$, if the patient is stable with normal chest $\mathrm{X}$ ray and acceptable HB.

\section{Expert opinion 7: Paolo Ferrari}

According to our program, we remove the chest drain in patients underwent lobectomies or segmentectomies, when a not bloody or chylous pleural fluid $<4 \mathrm{ml} / \mathrm{kg}$ have been collected in the last 24 hours. Moreover, no air leaks or a digital air leak quantification $<20 \mathrm{~mL} / \mathrm{min}$ must be respected.

\section{Expert opinion 8: Roberto Crisci}

I prefer to remove the drain for a quantity of serum $<200 \mathrm{~mL} / \mathrm{h}$.

\section{Expert opinion 9: Anthony M. H. Ho}

Chest drains are removed if drainage is $300 \mathrm{~mL} / 24$ hours. We consider over $300 \mathrm{~mL} / 24 \mathrm{~h}$ excessive and chest drain is 
usually kept in.

There are guidelines recommending that, for patients at high risk of VTE after surgery, continuous use of low-molecular-weight heparin for up to 4 weeks may be considered for long-term prevention. Is it feasible in clinical settings? Can physical prevention alone achieve the same effectiveness after such patients are discharged?

\section{Expert opinion 1: Alan D. L. Sihoe}

It is common knowledge (though not well documented in the literature) that Chinese patients have much lower risk of VTE than Caucasian patients. Therefore, those international guidelines may not be so suitable for Chinese patients. Over the last 20 years, we do not routinely use LMWH for any Chinese patients (we only use if patients have a known history of previous VTE). We use leg compression during surgery, and then TED stockings after surgery until patients are mobilizing well. Patients are fully mobile by the time of discharge, so we advise that patients can stop using TED stockings provided they ensure adequate mobilization after discharge. With this protocol, I have witnessed only 2 cases of VTE over the last 15 years.

\section{Expert opinion 2: Andrea Droghetti}

We use LWM Heparin for 2 weeks after discharge in high risk pts.

\section{Expert opinion 3: Antonio D'Andrilli}

In our opinion, due to early mobilization of patients in ERAS pathways, the postoperative period of LMW heparin administration can be shortened.

\section{Expert opinion 4: Calvin $\mathbf{N g}$}

Many of our patients with malignancy, and some with limited mobility or obese are the populations at high risk of developing VTE events. Some studies have shown lower incidence of subclinical DVT or PE when patients are given low-molecular-weight heparin in the early postoperative period, but not translating into VTE associated clinically significant event or mortality. Thus the real value of giving heparin in unknown. In our centre, we do not routinely give low-molecular -weight heparin to our lung surgery patients. We encourage early mobilization, and leg exercises even when they are lying on the bed or sitting out.

\section{Expert opinion 5: Luca Voltolini}

We believe that, for patients at high risk of VTE after surgery, continuous use of low-molecular-weight heparin for up to 4 weeks, is feasible and effective, even if there are no definitive data in the literature.

\section{Expert opinion 6: Majed Refai}

Physical prevention is not sufficient for oncological patients. We use single shot/day of low-molecular-weight heparin.

\section{Expert opinion 7: Paolo Ferrari}

Routinely use of LMWH in the first 30 days after surgery as VTE prevention is a feasible treatment in the clinical settings. Moreover, the physical prevention alone is patient dependent and should not fit adequate with a prophylaxis intent.

\section{Expert opinion 8: Roberto Crisci}

We use this scheme and do not believe that physical prevention alone can achieve the same efficacy.

\section{Expert opinion 9: Anthony M. H. Ho}

For patients at high risk of VTE (e.g., those with cancer), they are sent home to self-administer LMWH for 4 weeks. This is important as pulmonary embolus is usually fatal.

\section{Who needs airway preparation before a lung cancer surgery? How long does airway preparation take and what are the criteria?}

\section{Expert opinion 1: Alan D. L. Sihoe}

From a surgeon's perspective, we do not request any airway preparation for a standard lung cancer operation. If a patient is deemed to have a 'difficult airway' for intubation or if awake intubation is indicated, the in-charge anesthetist may use airway preparation as he/she wishes. However, this is an anesthetic-not surgical-issue, and hence it is not something that a surgeon would make the decision about.

\section{Expert opinion 2: Andrea Droghetti}

All patients start FKT when they start pathway and continue after surgery.

\section{Expert opinion 3: Antonio D'Andrilli}

Patients with severe COPD should undergo preop airway preparation. Two weeks of airway preparation are generally sufficient. 


\section{Expert opinion 4: Calvin $\mathrm{Ng}$}

Those patients with borderline pulmonary function, or low post-operative predicted lung capacity would be candidates for airway preparation. Patients with poorly controlled asthma or COPD are also candidates. We aim to optimize the high-risk patients for surgery to reduce respiratory complications.

\section{Expert opinion 5: Luca Voltolini}

We consider prehabilitation which includes aerobic training and respiratory exercises in patients with borderline respiratory function or exercise capacity. The median duration is 3 weeks, but no firm recommendations can be made on the exact nature of the intervention regarding frequency and duration.

\section{Expert opinion 6: Majed Refai}

All patients should meet the Physiotherapist at the moment of counselling and begin education before surgery. Severe COPD you have to optimize their treatment but you must not exceed 1 month to surgical treatment from the day of diagnosis. And repeat PFT and Chest Xray if necessary, otherwise the patients are not fit for ERAS (if they are > ASA 3).

\section{Expert opinion 7: Paolo Ferrari}

In our Institution all patients awaiting anatomical resections receive a respiratory physiotherapy assessment. According to preoperative PFTs and sputum collection microbiology results, antibiotics, glucocorticoids, bronchodilators, and mucolytic agents are administered. Patients with FEV1 $<60 \%$ are followed by the physiotherapy department for a tailored rehab program before surgery.

\section{Expert opinion 8: Roberto Crisci}

I think patients with chronic bronchopneumopathies need to be prepared. This preparation must be at least 10 days before the intervention.

\section{Expert opinion 9: Anthony M. H. Ho}

When infection is identified, the case is postponed or the problem treated. Chest physiotherapy and incentive spirometry as alluded to in the text of this manuscript are also used periperatively.

\section{Should anesthesia with spontaneous ventilation be included in the ERAS process?}

Expert opinion 1: Alan D. L. Sihoe

I do use GA with spontaneous ventilation ('non-intubated'
GA) in some patients. However, I do this out of academic interest to explore its potential-NOT because I am convinced it has any advantages at all. The current evidence for GA with spontaneous ventilation is quite poor, as the few studies reporting on this technique have small cohorts, limited study designs, and/or other methodological shortcomings. I would conclude that there is insufficient data to suggest that GA with spontaneous ventilation should be included in any ERAS protocol at the present time.

\section{Expert opinion 2: Andrea Droghetti}

That's sure... non intubated thoracic surgery must be investigated.

\section{Expert opinion 3: Antonio D'Andrilli}

In my opinion there are still not sufficient data and evidences to include anesthesia with spontaneous ventilation in the ERAS process.

\section{Expert opinion 4: Calvin $\mathrm{Ng}$}

There is probably sufficient evidence that in select patient populations, performing surgery with spontaneous ventilation could be beneficial, and can enhance recovery. However, I think we are far from putting it as a recommendation or essential part of ERAS, given the differences in local practices and skills. The concept of spontaneous ventilation could be included in the ERAS process.

\section{Expert opinion 5: Luca Voltolini}

No, or at least not yet.

\section{Expert opinion 6: Majed Refai}

Spontaneous ventilation (non intubated) may be included but we have to standardize the procedure.

\section{Expert opinion 7: Paolo Ferrari}

Although several studies report advantages of non-intubated thoracic surgery compared to general anesthesia with double lumen tube, the effect of spontaneous ventilation over surgical outcomes in lung cancer patients must be further evaluated, and should be avoided inside ERAS protocol.

\section{Expert opinion 8: Roberto Crisci}

Yes.

\section{Expert opinion 9: Anthony M. H. Ho}

No. Spontaneous ventilation is not possible in an open chest. 


\section{Conclusions}

ERAS is a multidisciplinary collaborative process that integrates a series of effective measures. In addition to the inputs of surgeons, anesthesiologists, rehabilitation therapists, and nursing staff, it also requires the active participation of patients and their families. It is hoped that the consensus on the perioperative management, including preoperative preparations (e.g., patient education, smoking cessation, preoperative pulmonary function assessment and airway management, and antiinfective treatment), intraoperative management (e.g., anesthesia and surgical management), and postoperative treatment (e.g., postoperative airway management and pulmonary rehabilitation, rational use of analgesics, and early ambulation) could help to put existing knowledge into practice in perioperative management and encourage the wider application ERAS in lung surgery.

\section{Acknowledgments}

None.

\section{Footnote}

Conflicts of Interest: The authors have no conflicts of interest to declare.

Ethical Statement: The authors are accountable for all aspects of the work in ensuring that questions related to the accuracy or integrity of any part of the work are appropriately investigated and resolved.

\section{References}

1. Kehlet H. Multimodal approach to control postoperative pathophysiology and rehabilitation. Br J Anaesth 1997;78:606-17.

2. Kehlet H, Wilmore DW. Evidence-based surgical care and the evolution of fast-track surgery. Ann Surg 2008;248:189-98.

3. Gonzalez M, Abdelnour-Berchtold E, Perentes JY, et al. An enhanced recovery after surgery program for videoassisted thoracoscopic surgery anatomical lung resections is cost-effective. J Thorac Dis 2018;10:5879-88.

4. Brown JK, Singh K, Dumitru R, et al. The Benefits of Enhanced Recovery After Surgery Programs and Their Application in Cardiothoracic Surgery. Methodist Debakey
Cardiovasc J 2018;14:77-88.

5. French DG, Plourde M, Henteleff H, et al. Optimal management of postoperative parenchymal air leaks. J Thorac Dis 2018;10:S3789-98.

6. Ni X, Jia D, Chen Y, et al. Is the Enhanced Recovery After Surgery (ERAS) Program Effective and Safe in Laparoscopic Colorectal Cancer Surgery? A Meta-Analysis of Randomized Controlled Trials. J Gastrointest Surg 2019;23:1502-12.

7. Stethen TW, Ghazi YA, Heidel RE, et al. Walking to recovery: the effects of missed ambulation events on postsurgical recovery after bowel resection. J Gastrointest Oncol 2018;9:953-961.

8. Laganà AS, Garzon S, Casarin J, et al. Acupoint Massage to Manage Postoperative Ileus in Gynecologic Laparoscopy: A New Potential Player in the Enhanced Recovery After Surgery (ERAS) Pathways? J Invest Surg 2019:1-3. [Epub ahead of print].

9. Keil DS, Schiff LD, Carey ET, et al. Predictors of Admission After the Implementation of an Enhanced Recovery After Surgery Pathway for Minimally Invasive Gynecologic Surgery. Anesth Analg 2019;129:776-83.

10. Ding $Y, G a o ~ Z$, Sun $Z$, et al. Enhanced recovery program in liver resection surgery: a single center experience. Transl Cancer Res 2018;7:1112-21.

11. Damania R, Cocieru A. Impact of enhanced recovery after surgery protocols on postoperative morbidity and mortality in patients undergoing routine hepatectomy: review of the current evidence. Ann Transl Med 2017;5:341.

12. Offodile AC 2nd, Gu C, Boukovalas S, et al. Enhanced recovery after surgery (ERAS) pathways in breast reconstruction: systematic review and meta-analysis of the literature. Breast Cancer Res Treat 2019;173:65-77.

13. Sharif-Askary B, Hompe E, Broadwater G, et al. The Effect of Enhanced Recovery after Surgery Pathway Implementation on Abdominal-Based Microvascular Breast Reconstruction. J Surg Res 2019;242:276-85.

14. Lin C, Wan F, Lu Y, et al. Enhanced recovery after surgery protocol for prostate cancer patients undergoing laparoscopic radical prostatectomy. J Int Med Res 2019;47:114-21.

15. Sugi M, Matsuda T, Yoshida T, et al. Introduction of an Enhanced Recovery after Surgery Protocol for RobotAssisted Laparoscopic Radical Prostatectomy. Urol Int 2017;99:194-200.

16. Dietz N, Sharma M, Adams S, et al. Enhanced Recovery After Surgery (ERAS) for Spine Surgery: A Systematic Review. World Neurosurg 2019;130:415-26. 
17. Angus M, Jackson K, Smurthwaite G, et al. The implementation of enhanced recovery after surgery (ERAS) in complex spinal surgery. J Spine Surg 2019;5:116-23.

18. Corniola MV, Debono B, Joswig H, et al. Enhanced recovery after spine surgery: review of the literature. Neurosurg Focus 2019 1;46:E2.

19. Caso R, Watson TJ, Khaitan PG, et al. Outcomes of minimally invasive sleeve resection. J Thorac Dis 2018;10:6653-9.

20. Chiou YW, Ting CK, Wang HY, et al. Enhanced recovery after surgery: Prediction for early extubation in videoassisted thoracic surgery using a response surface model in anesthesia. J Formos Med Assoc 2019;118:1450-7.

21. Pischik VG, Kovalenko A. The role of indocyanine green fluorescence for intersegmental plane identification during video-assisted thoracoscopic surgery segmentectomies. J Thorac Dis 2018;10:S3704-11.

22. Boffa DJ, Kosinski AS, Furnary AP, et al. Minimally Invasive Lung Cancer Surgery Performed by Thoracic Surgeons as Effective as Thoracotomy. J Clin Oncol 2018;36:2378-85.

23. Mayor MA, Khandhar SJ, Chandy J, et al. Implementing a thoracic enhanced recovery with ambulation after surgery program: key aspects and challenges. J Thorac Dis 2018;10:S3809-14.

24. D'Andrilli A, Rendina EA. Enhanced recovery after surgery (ERAS) and fast-track in video-assisted thoracic surgery (VATS) lobectomy: preoperative optimisation and care-plans. J Vis Surg 2018 5;4:4.

25. Batchelor TJP, Rasburn NJ, Abdelnour-Berchtold E et al. Guidelines for enhanced recovery after lung surgery: recommendations of the Enhanced Recovery After Surgery (ERAS@) Society and the European Society of Thoracic Surgeons (ESTS). Eur J Cardiothorac Surg 2019;55:91-115.

26. Patel B, Gauvin R, Absar S, et al. Computational and bioengineered lungs as alternatives to whole animal, isolated organ, and cell-based lung models. Am J Physiol Lung Cell Mol Physiol 2012;303:L733-47.

27. Pedoto A. Lung physiology and obesity: anesthetic implications for thoracic procedures. Anesthesiol Res Pract 2012;2012:154208.

28. Azharuddin S, Yunyongying P. Predicting the Risk of Postoperative Pulmonary Complications. Am Fam Physician 2019;100:499-501.

29. Lai Y, Wang X, Li P, et al. Preoperative peak expiratory flow (PEF) for predicting postoperative pulmonary complications after lung cancer lobectomy: a prospective study with 725 cases. J Thorac Dis 2018;10:4293-301.

30. Lee JH, Bae JI, Jang YE, et al. Lung protective ventilation during pulmonary resection in children: a prospective, single-centre, randomised controlled trial. Br J Anaesth 2019;122:692-701.

31. Li P, Li J, Lai Y, et al. Perioperative changes of serum albumin are a predictor of postoperative pulmonary complications in lung cancer patients: a retrospective cohort study. J Thorac Dis 2018;10:5755-63.

32. Garutti I, De la Gala F, Piñeiro P, et al. Usefulness of combining clinical and biochemical parameters for prediction of postoperative pulmonary complications after lung resection surgery. J Clin Monit Comput 2019;33:1043-54.

33. Arslantas MK, Kara HV, Tuncer BB, et al. Effect of the amount of intraoperative fluid administration on postoperative pulmonary complications following anatomic lung resections. J Thorac Cardiovasc Surg 2015;149:314-20.

34. Whyte RI, Grant PD. Preoperative patient education in thoracic surgery. Thorac Surg Clin 2005;15:195-201.

35. Lussiez AD, Burdick S, Kodali S, et al. Internet Usage Trends in Thoracic Surgery Patients and Their Caregivers. J Cancer Educ 2017;32:91-6.

36. Martin LW, Sarosiek BM, Harrison MA, et al. Implementing a Thoracic Enhanced Recovery Program: Lessons Learned in the First Year. Ann Thorac Surg 2018;105:1597-604.

37. Nair AS, Naik V, Saifuddin MS, et al. An Observational Study for Knowing the Compliance of Patients Scheduled for Major Abdominal and Thoracic Cancer Surgeries in a Single Specialty Center. Anesth Essays Res 2018;12:552-4.

38. Graffigna G, Barello S, Bonanomi A, et al. Measuring patient engagement: development and psychometric properties of the Patient Health Engagement (PHE) scale. Front Psychol 2015;6:274.

39. Graffigna G, Barello S, Bonanomi A. The role of Patient Health Engagement Model (PHE-model) in affecting patient activation and medication adherence: A structural equation model. PLoS One 2017;12:e0179865.

40. Graffigna G, Barello S. Spotlight on the Patient Health Engagement model (PHE model): a psychosocial theory to understand people's meaningful engagement in their own health care. Patient Prefer Adherence 2018;12:1261-71.

41. Smetana GW. Preoperative pulmonary evaluation. N Engl J Med 1999;340:937-44.

42. Nakagawa M, Tanaka H, Tsukuma H, et al. Relationship between the duration of the preoperative smoke-free 
period and the incidence of postoperative pulmonary complications after pulmonary surgery. Chest 2001;120:705-10

43. Mei J, Liu L, Tang M, et al. Airway bacterial colonization in patients with non-small cell lung cancer and the alterations during the perioperative period. J Thorac Dis 2014;6:1200-8.

44. Lugg ST, Tikka T, Agostini PJ, et al. Smoking and timing of cessation on postoperative pulmonary complications after curative-intent lung cancer surgery. J Cardiothorac Surg 2017 19;12:52.

45. Hanagiri T, Sugio K, Mizukami M, et al. Significance of Smoking as a Postoperative Prognostic Factor in Patients with Non-small Cell Lung Cancer. J Thorac Oncol 2008;3:1127-32.

46. Groth SS, Whitson BA, Kuskowski MA, et al. Impact of preoperative smoking status on postoperative complication rates and pulmonary function test results 1-year following pulmonary resection for non-small cell lung cancer. Lung Cancer 2009;64:352-7.

47. Bobbio A, Chetta A, Carbognani P, I et al. Changes in pulmonary function test and cardio-pulmonary exercise capacity in COPD patients after lobar pulmonary resection. Eur J Cardiothorac Surg 2005;28:754-8.

48. Dempsey TM, Scanlon PD. Pulmonary Function Tests for the Generalist: A Brief Review. Mayo Clin Proc 2018;93:763-771.

49. Ferguson MK, Vigneswaran WT. Diffusing capacity predicts morbidity after lung resection in patients without obstructive lung disease. Ann Thorac Surg 2008;85:115864; discussion 1164-5.

50. Brunelli A. Algorithm for functional evaluation of lung resection candidates: time for reappraisal? Respiration 2009;78:117-8.

51. Zhang R, Lee SM, Wigfield C, et al. Lung function predicts pulmonary complications regardless of the surgical approach. Ann Thorac Surg 2015;99:1761-7.

52. Yoshimi K, Oh S, Suzuki K, et al. Impact of Airflow Limitation on Comorbidities and Postoperative Complications in Patients Undergoing Thoracic Surgery: A Retrospective Observational Study. Ann Thorac Cardiovasc Surg 2016;22:146-52.

53. Chen YQ, Li JP, Xiao J. Prophylactic effectiveness of budesonide inhalation in reducing postoperative throat complaints. Eur Arch Otorhinolaryngol 2014;271:1667-72.

54. Zhang Y, Lou H, Wang Y, et al. Comparison of Corticosteroids by 3 Approaches to the Treatment of
Chronic Rhinosinusitis With Nasal Polyps. Allergy Asthma Immunol Res 2019;11:482-497.

55. Tecklin JS, Holsclaw DS Jr. Bronchial drainage with aerosol medications in cystic fibrosis. Phys Ther 1976;56:999-1003.

56. Sanchez-Lorente D, Navarro-Ripoll R, Guzman R, et al. Prehabilitation in thoracic surgery. J Thorac Dis 2018;10:S2593-S2600.

57. Dai L, Kang X, Yan W, et al. Analysis of Prolonged Hospitalizations (Longer than 7 days): 115 Lung Cancer Patients after Video Assistant Thoracic Surgery (VATS). Zhongguo Fei Ai Za Zhi 2018;21:223-9.

58. Chiou YW, Ting CK, Wang HY, et al. Enhanced recovery after surgery: Prediction for early extubation in videoassisted thoracic surgery using a response surface model in anesthesia. J Formos Med Assoc 2019;118:1450-7.

59. Pauli H, Eladawy M, Park J. Anesthesia for robotic thoracic surgery. Ann Cardiothorac Surg 2019;8:263-8.

60. Arslantas MK, Kara HV, Tuncer BB, et al. Effect of the amount of intraoperative fluid administration on postoperative pulmonary complications following anatomic lung resections. J Thorac Cardiovasc Surg 2015;149:31420, 321.e1.

61. Asteriou C, Lazopoulos A, Rallis T, et al. Video-assisted thoracic surgery reduces early postoperative stress. A single-institutional prospective randomized study. Ther Clin Risk Manag 2016;12:59-65.

62. Zheng M, Niu Z, Chen P, et al. Effects of bronchial blockers on one-lung ventilation in general anesthesia: A randomized controlled trail. Medicine (Baltimore) 2019;98:e17387.

63. Ishikawa S, Yoshida I, Ohtaki A, et al. Successful surgical management of emphysematous giant bullae accompanied by severe bronchial asthma: report of a case. Surg Today 1995;25:560-2.

64. Franceschini F, De Benedictis FM, Peroni DG, et al. Anesthesia in children with asthma and rhinitis. Int J Immunopathol Pharmacol 2011;24:S83-90.

65. L'her E, Jaber S, Verzilli D, et al. Automated oxygen administration versus conventional oxygen therapy after major abdominal or thoracic surgery: study protocol for an international multicentre randomised controlled study. BMJ Open 2019;9:e023833.

66. Bernasconi F, Piccioni F. One-lung ventilation for thoracic surgery: current perspectives. Tumori 2017;103:495-503.

67. Lohser J, Slinger P. Lung Injury After One-Lung Ventilation: A Review of the Pathophysiologic Mechanisms Affecting the Ventilated and the Collapsed Lung. Anesth 
Analg 2015;121:302-18.

68. Güldner A, Kiss T, Serpa Neto A, et al. Intraoperative protective mechanical ventilation for prevention of postoperative pulmonary complications: a comprehensive review of the role of tidal volume, positive end-expiratory pressure, and lung recruitment maneuvers. Anesthesiology 2015;123:692-713.

69. Licker M, Fauconnet P, Villiger Y, Tschopp JM. Acute lung injury and outcomes after thoracic surgery. Curr Opin Anaesthesiol 2009;22:61-7.

70. Kansier N, Varghese TK Jr, Verrier ED, et al. Accreditation Council for Graduate Medical Education case log: general surgery resident thoracic surgery experience. Ann Thorac Surg 2014;98:459-64; discussion 464-5.

71. Hung MH, Hsu HH, Chen JS, et al. Arts on tailored postoperative analgesia after video-assisted thoracoscopic surgery. Video-assist Thorac Surg 2019;4:11.

72. Bertolaccini L, Viti A, Cavallo A, et al. Results of Li-Tho trial: a prospective randomized study on effectiveness of

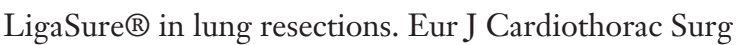
2014;45:693-8; discussion 698.

73. Moritz A, Schmidt J, Schreiner W, et al. Combined recurrent laryngeal nerve monitoring and one-lung ventilation using the EZ-Blocker and an electromyographic endotracheal tube. J Cardiothorac Surg 2019 19;14:111.

74. Mehta HJ, Malhotra P, Begnaud A, et al. Treatment of alveolar-pleural fistula with endobronchial application of synthetic hydrogel. Chest 2015;147:695-9.

75. Bakhos CT, Wang SC, Rosen JM. Contemporary role of minimally invasive thoracic surgery in the management of pulmonary arteriovenous malformations: report of two cases and review of the literature. J Thorac Dis 2016;8:195-7.

76. Scarpino M, Lanzo G, Cappelli F, et al. Cerebral Fat Embolism After Video-Assisted Thoracic Surgery. Ann Thorac Surg. 2016;102:e409-11.

77. Li G, Wang JS, Qin SD, et al. Clinical study of the oxygen drive aerosol inhalation with budesonide and ambroxol in the prevention of adult post-thoracotomy pneumonia. Beijing Da Xue Xue Bao Yi Xue Ban 2018;50:840-4.

78. Faisy C, Grassin-Delyle S, Blouquit-Laye S, et al. Wnt/ $\beta$-catenin signaling modulates human airway sensitization induced by $\beta 2$-adrenoceptor stimulation. PLoS One 2014 31;9:e111350.

79. Reeve J, Denehy L, Stiller K. The physiotherapy management of patients undergoing thoracic surgery: a survey of current practice in Australia and New Zealand.
Physiother Res Int 2007;12:59-71.

80. Beach L, Denehy L, Lee A. The efficacy of minitracheostomy for the management of sputum retention: a systematic review. Physiotherapy 2013;99:271-7.

81. Liu CJ, Tsai WC, Chu CC, et al. Is incentive spirometry beneficial for patients with lung cancer receiving videoassisted thoracic surgery? BMC Pulm Med 2019 8;19:121.

82. Tomaszek L, Fenikowski D, Komotajtys H, et al. Ropivacaine/Fentanyl vs. Bupivacaine/Fentanyl for Pain Control in Children after Thoracic Surgery: A Randomized Study. Pain Manag Nurs 2019;20:390-7.

83. Dominguez DA, Ely S, Bach C, et al. Impact of intercostal nerve blocks using liposomal versus standard bupivacaine on length of stay in minimally invasive thoracic surgery patients. J Thorac Dis 2018;10:6873-9.

84. Haager B, Schmid D, Eschbach J, et al. Regional versus systemic analgesia in video-assisted thoracoscopic lobectomy: a retrospective analysis. BMC Anesthesiol 2019;19:183.

85. Simeoforidou M, Vretzakis G, Bareka M, et al. Thoracic epidural analgesia with levobupivacaine for 6 postoperative days attenuates sympathetic activation after thoracic surgery. J Cardiothorac Vasc Anesth 2011;25:817-23.

86. Bauer C, Hentz JG, Ducrocq X, et al. Lung function after lobectomy: a randomized, double-blinded trial comparing thoracic epidural ropivacaine/sufentanil and intravenous morphine for patient-controlled analgesia. Anesth Analg 2007;105:238-44.

87. Erden S, Senol Celik S. The effect of transcutaneous electrical nerve stimulation on post-thoracotomy pain. Contemp Nurse 2015;51:163-70.

88. Engen DJ, Carns PE, Allen MS, et al. Evaluating efficacy and feasibility of transcutaneous electrical nerve stimulation for postoperative pain after videoassisted thoracoscopic surgery: A randomized pilot trial. Complement Ther Clin Pract 2016;23:141-8.

89. Chen G, Li Y, Zhang Y, et al. Effects of serratus anterior plane block for postoperative analgesia after thoracoscopic surgery compared with local anesthetic infiltration: a randomized clinical trial. J Pain Res 2019;12:2411-7.

90. Abdallah NM, Bakeer AH, Youssef RB, et al. Ultrasoundguided continuous serratus anterior plane block: dexmedetomidine as an adjunctive analgesic with levobupivacaine for post-thoracotomy pain. A prospective randomized controlled study. J Pain Res 2019;12:1425-31.

91. Kuroda H, Sugita Y, Watanabe K, et al. Successful postoperative recovery management after thoracoscopic 
lobectomy and segmentectomy using an ERASbased protocol of immediate ice cream intake and early ambulation: a 3-year study. Cancer Manag Res 2019;11:4201-7.

92. Leithauser DJ. Rational principles of early ambulation. J Int Coll Surg 1949;12:368-74.

93. Leithauser DJ, Saraf L, Smyka S, et al. Prevention of embolic complications from venous thrombosis after surgery; standardized regimen of early ambulation. J Am Med Assoc 1951;147:300-3.

94. Leithauser DJ, Saraf L, Smyka S, et al. Early ambulation in prevention of postoperative thromboembolism;supplementary clinical note. J Am Med Assoc 1952;149:268-9.

95. Kendall F, Abreu P, Pinho P, et al. The role of

Cite this article as: Gao S, Barello S, Chen L, Chen C, Che G, Cai K, Crisci R, D’Andrilli A, Droghetti A, Fu X, Ferrari PA, Fernando HC, Ge D, Graffigna G, Huang Y, Hu J, Jiao W, Jiang G, Li X, Li H, Li S, Liu L, Ma H, Ma D, Martinez G, Maurizi G, Phan K, Qiao K, Refai M, Rendina EA, Shao G, Shen J, Tian H, Voltolini L, Vannucci J, Vanni C, Wu Q, Xu S, Yu F, Zhao S, Zhang P, Zhang L, Zhi X, Zhu C, Ng C, Sihoe ADL, Ho AMH. Clinical guidelines on perioperative management strategies for enhanced recovery after lung surgery. Transl Lung Cancer Res 2019;8(6):1174-1187. doi: 10.21037/tlcr.2019.12.25 physiotherapy in patients undergoing pulmonary surgery for lung cancer. A literature review. Rev Port Pneumol (2006) 2017;23:343-51.

96. Bhatt NR, Sheridan G, Connolly M, et al. Postoperative exercise training is associated with reduced respiratory infection rates and early discharge: A case-control study. Surgeon 2017;15:139-46.

97. Quist M, Sommer MS, Vibe-Petersen J, et al. Early initiated postoperative rehabilitation reduces fatigue in patients with operable lung cancer: A randomized trial. Lung Cancer. 2018;126:125-32.

98. Jonsson M, Hurtig-Wennlöf A, Ahlsson A, et al. Inhospital physiotherapy improves physical activity level after lung cancer surgery: a randomized controlled trial. Physiotherapy 2019;105:434-41. 